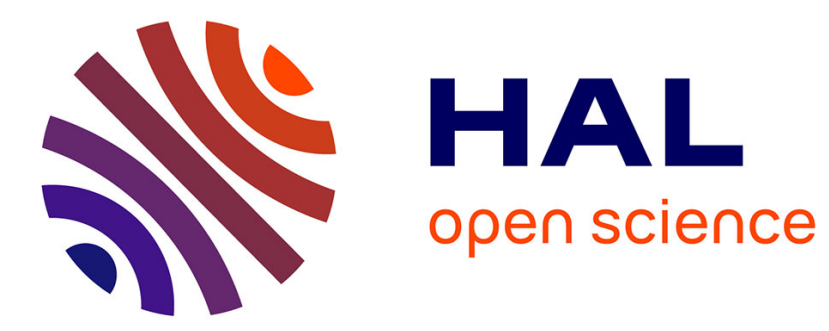

\title{
Infection de prothèse vasculaire : 18TEP-FDG vs scintigraphie aux leucocytes marqués (planaires et TEMP/TDM)
}

\author{
C. Agius, Hervé Rakotonirina, Franck Lacœuille, Francis Bouchet, Laurent \\ Vervueren, Jean-Jacques Le Jeune, Olivier-François Couturier
}

\section{To cite this version:}

C. Agius, Hervé Rakotonirina, Franck Lacœuille, Francis Bouchet, Laurent Vervueren, et al.. Infection de prothèse vasculaire : 18TEP-FDG vs scintigraphie aux leucocytes marqués (planaires et TEMP/TDM). Médecine Nucléaire - Imagerie Fonctionnelle et Métabolique, 2011, 35 (12), Non spécifié. 10.1016/j.mednuc.2011.09.004 . hal-03179483

\section{HAL Id: hal-03179483 \\ https://univ-angers.hal.science/hal-03179483}

Submitted on 24 Mar 2021

HAL is a multi-disciplinary open access archive for the deposit and dissemination of scientific research documents, whether they are published or not. The documents may come from teaching and research institutions in France or abroad, or from public or private research centers.
L'archive ouverte pluridisciplinaire HAL, est destinée au dépôt et à la diffusion de documents scientifiques de niveau recherche, publiés ou non, émanant des établissements d'enseignement et de recherche français ou étrangers, des laboratoires publics ou privés. 


\title{
Infection de prothèse vasculaire : ${ }^{18}$ TEP-FDG vs scintigraphie aux leucocytes marqués (planaires et TEMP/TDM)
}

\author{
Vascular prosthesis infection: Comparison between ${ }^{18}$ FDG-PET and labeled leukocytes \\ scintigraphy (planar and SPECT-CT)
}

\author{
C. Agius ${ }^{a}$, H. Rakotonirina ${ }^{\text {a }}$, F. Lacoeuille ${ }^{\mathrm{b}}$, F. Bouchet ${ }^{\mathrm{a}}$, L. Vervueren ${ }^{\mathrm{a}}$, J.-J. Le Jeune ${ }^{\mathrm{a}}$, O. Couturier ${ }^{\mathrm{a}, *}$ \\ ${ }^{a}$ Service de médecine nucléaire, département de médicine nucléaire, CHU d'Angers, 4, rue Larrey, 49933 Angers cedex 9, France \\ ${ }^{\mathrm{b}}$ Service de radiopharmacie, $\mathrm{CHU}$ d'Angers, Angers, France \\ Reçu le 5 avril 2011 ; accepté le 28 septembre 2011 \\ Disponible sur Internet le 23 novembre 2011
}

\begin{abstract}
Résumé
L'infection de prothèse vasculaire est une complication rare, mais grave. Son diagnostic est difficile, notamment car l'imagerie conventionnelle est peu spécifique. Le but de cette étude préliminaire était de comparer l'apport diagnostique de la tomographie par émission de positons au ${ }^{18}$ FDG (TEP- ${ }^{18} \mathrm{FDG}$ ) à celui de la scintigraphie aux leucocytes marqués à l'HMPAO- ${ }^{99 \mathrm{~m}} \mathrm{Tc}$ dans cette indication. Réalisation prospective d'une TEP- ${ }^{18} \mathrm{FDG}$ couplée au scanner et d'une scintigraphie aux leucocytes marqués (planaires à la sixième et $24^{\mathrm{e}}$ heures + tomographie par émission monophotonique couplée à la tomodensitométrie [TEMP-TDM] à la sixième heure) chez 11 patients (22 prothèses au total avec suspicion clinique d'infection pour 14 d'entre elles). Relecture rétrospective, en aveugle, des examens par deux médecins nucléaires. Interprétation basée sur une analyse visuelle de l'intensité et de l'aspect de la fixation des prothèses. L'étalon de référence était la bactériologie ou un suivi supérieur à six mois. L'infection de prothèse était retenue dans huit cas. La TEP retrouvait huit vrais positifs et un faux positif et la scintigraphie, huit vrais positifs et aucun faux positif. Une hyperfixation focale ou hétérogène supérieure ou égale à la fixation hépatique était retenue comme positive en TEP. Une hyperfixation focale stable ou augmentée à 24 heures était retenue comme positive pour la scintigraphie. La TEMP-TDM a permis de localiser avec précision les infections limitées aux tissus mous. La TEP- ${ }^{18} \mathrm{FDG}$ pourrait être effectuée en première intention dans les suspicions d'infection de prothèses vasculaires et complétée, en cas de doute, par une scintigraphie aux leucocytes avec TEMP-TDM.
\end{abstract}

(C) 2011 Elsevier Masson SAS. Tous droits réservés.

Mots clés : Infection de prothèse vasculaire ; TEP-TDM au ${ }^{18} \mathrm{FDG}$; Scintigraphie aux leucocytes marqués à l'HMPAO- ${ }^{99 \mathrm{~m}} \mathrm{Tc}$; TEMP-TDM

\begin{abstract}
Vascular prosthesis infection is an uncommon but life-threatening complication. Its diagnosis is difficult to establish especially due to the low specificity of computed tomography (CT). The aim of this preliminary study was to compare the diagnostic value of positron emission tomography with ${ }^{18}$ FDG $\left({ }^{18}\right.$ FDG-PET) and ${ }^{99 \mathrm{~m}}$ Tc-HMPAO-labeled leukocytes scintigraphy in this indication. ${ }^{18}$ FDG-PET/CT and ${ }^{99 \mathrm{~m}} \mathrm{Tc}-\mathrm{HMPAO}-\mathrm{labeled}$ leukocytes scintigraphy (planar at 6th and 24th hours after injection + SPECT/CT at the 6th hour) were prospectively performed in 11 patients (total of 22 vascular prosthesis with 14 clinical suspicions of infection). Both scans were retrospectively and blindly assessed by two independent nuclear medicine physicians. Interpretation was based on visual analysis. The gold standard was bacteriology findings or clinical follow-up greater than 6 months. Eight prostheses were considered as infected. PET found eight true-positive and one false-positive. Scintigraphy found eight true-positive and no false-positive. A focal or heterogeneous FDG-uptake higher or equal than hepatic uptake was considered as positive in PET. A focal prosthetic activity, stable or increased at the 24th hour was considered as positive in labeled leukocyte scintigraphy. SPECT/CT gave accurate anatomic localization and differentiated clearly infections of soft tissues from those of prostheses. ${ }^{18}$ FDG-PET could be performed in first-line in suspicion of vascular prosthesis infection. In litigious cases, $\mathrm{a}^{99 \mathrm{~m}} \mathrm{Tc}-\mathrm{HMPAO}-$ labeled leukocytes scintigraphy in association with SPECT/CT could bring additional arguments for infection diagnosis.
\end{abstract}

(C) 2011 Elsevier Masson SAS. All rights reserved.

Keywords: Vascular prosthesis infection; ${ }^{18}$ FDG-PET/CT; ${ }^{99 \mathrm{~m}} \mathrm{Tc}-\mathrm{HMPAO}-$ labeled leukocytes scintigraphy; SPECT-CT

\footnotetext{
* Auteur correspondant.

Adresse e-mail : OlCouturier@chu-angers.fr (O. Couturier).
} 


\section{Introduction}

L'infection de prothèse vasculaire (IPV) est une complication rare qui concerne 0,5 à $5 \%$ des prothèses vasculaires $(\mathrm{PV})$ [1]. Le délai de survenue des IPV est variable, de quelques mois à plusieurs années après la chirurgie [2]. Elle engendre une forte morbi-mortalité dans plus de $50 \%$ des cas, qui dépend de la localisation de la prothèse infectée : si elle est centrale, elle engage le pronostic vital; si elle est périphérique, le pronostic fonctionnel avec risque d'amputation. La morbidité dépend également de la nature du pathogène : les flores bactériennes les plus contaminantes sont celles à point de départ cutané ou digestif, expliquant le spectre bactérien où prédominent les staphylocoques (50\% Saphylococus aureus méthi-sensibles et $20 \%$ S. aureus méthi-résistants) et les entérobactéries [3]. L'IPV nécessite une prise en charge précoce et un traitement lourd chez ces patients souvent polyvasculaires [4-8].

Le diagnostic d'IPV est souvent difficile car les germes ne sont retrouvés que dans $50 \%$ des cas et l'imagerie conventionnelle est souvent non spécifique. En effet, le scanner hélicoïdal injecté, effectué en première intention peut montrer des bulles d'air périprothétiques (présentes dans $50 \%$ des cas en postopératoire), ainsi qu'une infiltration des tissus mous périprothétiques (seulement pour les IPV de haut grade). Les autres critères de positivité sont la présence d'un épaississement pariétal du greffon, d'un pseudo-anévrysme, d'une thrombose ou d'une collection liquidienne périprothétique. Le scanner présente ainsi une sensibilité estimée à $75 \%$ et une spécificité à 56,6\% dans l'IPV selon une méta-analyse réalisée par Annovazzi A. et al. [9]. Les diagnostics différentiels sont la présence d'un hématome, d'une fibrose ou simplement les remaniements postopératoires.

L'étalon de référence actuel en imagerie fonctionnelle de l'infection en général est la scintigraphie aux leucocytes marqués (SLM), avec une haute sensibilité $(97,7 \%)$ et une grande spécificité $(88,6 \%)$. Les premiers auteurs à avoir publié des grosses séries pour évaluer la SLM dans l'IPV sont Liberatore $\mathrm{M}$ et al. [10] et Prats E et al. [11]. Ils retrouvent une sensibilité de $100 \%$ et une spécificité de 92,5 et $100 \%$, respectivement. Les études de Filippi L et al. [12] et Bar Shalom R et al. [13] ont montré l'apport de la tomographie par émission monophotonique (TEMP) couplée à la tomodensitométrie (TDM) en complément des images planaires dans divers types d'infections (fièvre d'origine indéterminée, ostéomyélite, infection des tissus mous et IPV), qui permet une localisation anatomique précise des foyers hyperfixants. Cependant, l'utilisation de l'imagerie hybride dans la SLM a été peu explorée dans l'IPV (essentiellement quelques cas cliniques).

La tomographie par émission de positons au fluorodésoxyglucose (TEP-FDG), utilisée avant tout en oncologie, a montré un intérêt croissant dans le diagnostic et le suivi de maladies inflammatoires et dans les processus infectieux via l'augmentation de l'activité glycolytique des lymphocytes et des macrophages activés dans ces pathologies. Si la TEP-FDG semble moins performante dans les infections des prothèses orthopédiques que la SLM [14], elle est utile dans l'exploration d'une fièvre d'origine indéterminée [15] avec une contribution dans la littérature qui varie de 41 à $69 \%$ [16].

Plusieurs études depuis 2000 ont montré l'intérêt de la TEPFDG dans les IPV, mais la plupart avec des séries peu importantes [1,2,17].

L'objectif de notre étude est double :

- évaluer les performances diagnostiques de la TEP-FDG dans le diagnostic d'IPV et les comparer à celles de la SLM avec analyse TEMP-TDM ;

- proposer un protocole d'imagerie (arbre décisionnel) en routine en cas de suspicion d'IPV.

\section{Patients et méthode}

\subsection{Population de patients}

Onze patients (11 hommes, âge moyen : $65 \pm 10,6$ ans ; min : 47 ans, $\max$ : 81 ans) ont été explorés au CHU d'Angers pour suspicion d'IPV entre décembre 2006 et août 2009. Ces patients n'ont pas fait l'objet de critères d'inclusion ou de non inclusion particuliers et ont été adressés essentiellement par les services de maladies infectieuses et de chirurgie vasculaire.

Ces patients sont porteurs de $22 \mathrm{PV}$ au total, allant de une à quatre PV par patient (14 PV aorto- ou ilio-fémorales, trois PV fémoro-poplitées, quatre PV aortiques ou supra-aortiques et une PV de la veine cave inférieure (VCI) ; âge moyen : $26 \pm 41$ mois, $\min$ : un mois, $\max : 106$ mois). Les caractéristiques des patients et de leurs PV sont résumées dans le Tableau 1.

Quatorze sites prothétiques étaient suspects d'infection chez ces patients soit de façon directe (thrombose, abcès, écoulement de la cicatrice, fistule digestive ...), soit de façon indirecte (fièvre, contexte d'endocardite ou d'ostéite ...) (Tableau 2).

Les examens TEP-FDG et SLM ont été presque tous réalisés après l'instauration de l'antibiothérapie, le début de celle-ci par rapport à la date de réalisation de l'imagerie n'étant pas toujours précisé dans les dossiers patients et donc difficile à évaluer.

\subsection{Gold standard}

Le diagnostic d'IPV a été retenu :

- sur les données bactériologiques obtenues soit sur pièce opératoire après reprise chirurgicale, soit sur des prélèvements locaux ou des hémocultures ;

- en l'absence de données bactériologiques, sur les données cliniques (abcès périprothétique, fistulisation digestive), le suivi clinicobiologique (évolution sous antibiothérapie) et l'imagerie anatomique et fonctionnelle sur plus de six mois.

\subsection{Protocole d'imagerie}

Tous les patients ont bénéficié d'un examen TEP-FDG et d'une SLM dont l'ordre de réalisation a été aléatoire (délai 
Tableau 1

Caractéristiques des patients.

Patients' characteristics.

\begin{tabular}{|c|c|c|c|c|c|c|c|c|c|c|}
\hline Patients & Âge & Sexe & Contexte clinique & Diabète & $\begin{array}{l}\text { Nombre de prothèses } \\
\text { (localisation) }\end{array}$ & $\begin{array}{l}\text { Type de } \\
\text { prothèse }\end{array}$ & $\begin{array}{l}\text { Âge de la } \\
\text { prothèse [3] }\end{array}$ & CRP & $\mathrm{GB}\left(\mathrm{mm}^{3}\right)$ & Germes \\
\hline 1 & 80 & M & Thrombose de prothèse fébrile & Non & $2(\mathrm{IF} G+\mathrm{D})$ & DACRON & $2 \times 11$ & 89 & 6930 & SAMS \\
\hline 2 & 62 & M & Abcès du scarpa droit & Non & 1 (trépied fémoral D) & DACRON & 4 & 28 & 7690 & $\begin{array}{l}\text { Streptocoque } \\
\text { agalactiae }\end{array}$ \\
\hline 3 & 68 & M & Lâchage de sutures Lymphocèle fébrile & Non & $2(\mathrm{AoBF})$ & DACRON & $2 \times 3$ & 136 & 13560 & Non retrouvé \\
\hline 4 & 81 & M & Mal perforant plantaire surinfecté & Type 2 & 1 (FP G) & Polyester & 1 & 138 & 13470 & Non retrouvé \\
\hline 5 & 67 & M & Sigmoidite. Abcès iliaque G & Non & 2 (by-pass AoBF) & DACRON & $2 \times 106$ & 189 & 21000 & E. coli \\
\hline 6 & 61 & M & $\begin{array}{l}\text { abcès périprothétique }+ \text { embols } \\
\text { septiques cérébraux }\end{array}$ & Non & 3 (ISC,Ao thoracique, TABC) & Polyester & $3 \times 26$ & 164 & 10100 & Non retrouvé \\
\hline 7 & 56 & M & Chirurgie en urgence lymphocèle fébrile & Non & $2(\mathrm{AoBF})$ & Polyester & $2 \times 102$ & $<3$ & 6280 & Non retrouvé \\
\hline 8 & 65 & M & Endocardite thrombose prothèse & Type 1 & 2 (AoF D + iliaque G) & DACRON & $2 \times 93$ & 20 & 10700 & Non retrouvé \\
\hline 9 & 55 & M & Collections périprothétique fébriles & Non & $2(\mathrm{IBF})$ & DACRON & $2 \times 28$ & 35 & 4680 & Non retrouvé \\
\hline 10 & 75 & M & Ostéite sur ulcère artériel. septicémie & Type 2 & $1(\mathrm{FP} \mathrm{G})$ & Polyester & 59 & 32 & 6580 & SAMR \\
\hline 11 & 47 & M & Fistule prothétique sur péritonite & Non & $\begin{array}{l}4 \text { (Aorte sous-rénale, } \\
\text { VCI, AoBF) }\end{array}$ & Non connue & $4 \times 5$ & 205 & 14510 & $\begin{array}{l}\text { Streptocoque } \\
\text { anginosus }\end{array}$ \\
\hline
\end{tabular}

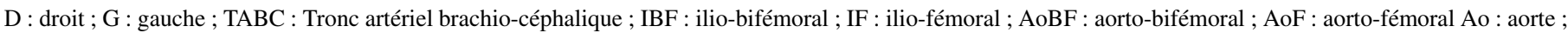
FP : fémoro-poplité ; ISC : inter-sous-clavier ; VCI : veine cave inférieure.

moyen entre les deux examens : huit jours ; min : un jour, max : 21 jours).

L'examen TEP a été réalisé sur un modèle Discovery ST de General Electric couplé à une TDM. Après six heures de jeun et 60 minutes de repos, une activité de $4 \mathrm{MBq} / \mathrm{kg}$ de ${ }^{18} \mathrm{FDG}$ (activité moyenne : 271,8 $\pm 51,3 \mathrm{MBq}$ ) a été injectée après contrôle de la glycémie $(1,18 \pm 0,25 \mathrm{~g} / \mathrm{L})$. Il a été injecté $20 \mathrm{mg}$ de Lasilix en intraveineux direct 30 minutes avant l'acquisition TEP afin de diminuer au maximum l'activité des voies urinaires. Il a ensuite été demandé au patient d'uriner avant l'acquisition. L'acquisition a été effectuée 60 minutes après l'injection en mode 3D par des pas de deux minutes 30 secondes depuis les membres inférieurs (de façon à couvrir entièrement le trajet de la ou des PV) jusqu'au sommet du crâne. Les paramètres de la TDM de repérage et de correction d'atténuation ont été les suivants : $80 \mathrm{mAs}, 120 \mathrm{kV}$. La reconstruction et l'analyse des images ont été effectuées sur une console de traitement Xéleris en utilisant des algorithmes de reconstruction itérative (OSEM : deux itérations et 30 sousgroupes).

Les SLM ont été réalisées sur la gamma-caméra hybride Hawkeye de General Electric. Le protocole a consisté en la réalisation d'images à la sixième heure (planaires + TEMP$\mathrm{TDM}$ ) et à la $24^{\mathrm{e}}$ heure (planaires) après l'injection d'une activité de $272 \pm 51 \mathrm{MBq}$ des leucocytes du patient marqués par le complexe HMPAO-Tc $99 \mathrm{~m}$ (Céretec ${ }^{\mathbb{R}}$ ). Les images planaires acquises en prétemps (dix minutes à la sixième heure et 20 minutes à la $24^{\mathrm{e}}$ heure) ont été centrées sur tout le trajet prothétique et l'acquisition tomoscintigraphique, sur le (les) foyer(s) hyperfixant(s) visualisé(s).

Les paramètres d'acquisitions en TEMP étaient pour la caméra Hawkeye : angles de trois degrés, 180 degrés par tête,

Tableau 2

Prothèses vasculaires et imagerie.

Vascular prostheses and imaging.

\begin{tabular}{clllr}
\hline Patient & site(s) suspect(s) d'infection (infection retenue) & TEP & SLM & Intervalle TEP/SLM (jours) \\
\hline 1 & G (oui) & Positif (VP) & Positif (VP) & 2 \\
2 & Scarpa D (non) & Négatif (VN) & Négatif (VN) & 5 \\
3 & Trépied fémoral D (non) & Négatif (VN) & Négatif (VN) & 3 \\
4 & G (oui) & Positif (VP) & Positif (VP) & 13 \\
5 & G (oui) & Positif (VP) & Positif (VP) & 2 \\
6 & Inter-sous-clavier (oui) & Positif (VP) & Positif (VP) & 7 \\
7 & Scarpa d et g (oui à d) & Positif D (VP), G (FP) & Positif D (VP), négatif G (VN) & 7 \\
8 & D + G (non) & Négatif G et D (VN), & Négatif D et G (VN) & 12 \\
9 & Scarpa D et G (oui D + G) & Positif D et G (VP) & 1 \\
10 & G (non) & Négatif (VN) & Négatif (VN) & 13 \\
11 & VCI (oui) & Positif (VP) & Positif (VP) & 21 \\
& Total : 14 sites suspectés & Total : 8 VP, 5 VN, 1 FP, 0 FN & Total : 8 VP, 6 VN, 0 FP, 0 VN
\end{tabular}

IBF : ilio-bifémoral ; D : droit ; G : gauche ; AoBF : aorto-bifémoral ; Ao : aorte ; FP : fémoro-poplité ; VCI : veine cave inférieure ; VP : vrai positif ; VN : vrai négatif ; FP : faux positif. 
40 secondes par pas ; matrice $128 \times 128$; zoom 1. Les paramètres TDM étaient : $140 \mathrm{kV}, 2,5 \mathrm{~mA}, 2,6$ rotations par minute, acquisition de type axiale de $5 \mathrm{~mm}$ coupes jointives, matrice $512 \times 512$; filtre de reconstruction standard aux normes du constructeur. La reconstruction et l'analyse des images ont été effectuées sur les consoles de traitement spécifiques aux constructeurs en utilisant des algorithmes de type OSEM (deux itérations et huit sous-ensembles).

\subsection{Technique de marquage et contrôle de qualité des leucocytes marqués}

\subsubsection{Principe}

La préparation des leucocytes marqués est réalisée par un radiopharmacien. Elle consiste à isoler et marquer les leucocytes du patient après un prélèvement de $100 \mathrm{~mL}$ de sang total en présence de $20 \%$ d'une solution anticoagulante de type ACD formule A. Le marquage du culot leucocytaire est quant à lui réalisé à l'aide d'un complexe lipophile technétié d'Hexaméthyl Propylène Amine Oxime (HMPAO : Céretec ${ }^{\circledR}$ ).

\subsubsection{Procédure}

Le marquage des leucocytes est réalisé dans une salle blanche (salle à empoussièrement contrôlé de classe Iso 7), dédiée aux marquages cellulaires. Les opérations de manipulation des cellules ont lieu sous hotte à flux laminaire. La sédimentation est réalisée sous incubateur à $37{ }^{\circ} \mathrm{C}$ (certains préconisent une sédimentation à température ambiante afin de diminuer le risque bactériologique [18]) pendant une durée de 45 min à à une heure 15 minutes afin d'obtenir un plasma riche en leucocytes et plaquettes. Le plasma est prélevé puis centrifugé (1000 RPM, cinq minutes), le culot leucocytaire est ainsi isolé puis mis en incubation dans une solution de ${ }^{99 \mathrm{~m}} \mathrm{Tc}$ HMPAO (700 MBq) pendant dix minutes. Après l'incubation le culot leucocytaire est lavé par du plasma sans cellule $(10 \mathrm{~mL})$ et centrifugé (1000 RPM, cinq minutes) afin de permettre le calcul du rendement de marquage. Après vérification du contrôle de qualité et validation de la préparation, une suspension leucocytaire marquée dans une seringue de 1,5 à $2,5 \mathrm{~mL}$ est injectée au patient (150 à $300 \mathrm{MBq}$ par patient).

\subsubsection{Contrôle qualité}

L'ensemble des contrôles qualités sont validés par un radiopharmacien.

2.4.3.1. Pureté radiochimique et rendement de marquage. La pureté radiochimique (PRC exprimée en \%) de $1^{999 \mathrm{~m}}$ Tc-HMPAO servant au marquage est vérifiée avant toute utilisation (PRC conforme $>90 \%$ ). Au cours de la procédure de marquage, le rendement de marquage $(\mathrm{R})$ est calculé de la manière suivante avec des normales comprises entre 40 et $55 \%$ :
2.4.3.2. Contrôle microscopique. Pour vérifier la bonne qualité de la préparation finale, il est réalisé en cellule de Malassez une numération leucocytaire (norme $>100,10^{6}$ leucocytes marqués), une étude de la pureté des leucocytes marques (norme $>90 \%$ ) et une étude la viabilité des leucocytes (norme $>90 \%$ ).

\subsection{Analyse des données d'imagerie TEP-FDG et SLM}

\subsubsection{Examen TEP-FDG}

L'interprétation a été basée sur l'analyse visuelle des zones d'hyperfixation quant à leur aspect (focale, diffuse ou mixte) et leur intensité (cotation en quatre niveaux $\geq 1$. faible : égale au BDF local, 2. intermédiaire : supérieure au BDF local et inférieure à la fixation hépatique, 3. modérée : égale à la fixation hépatique, 4. intense : supérieure à la fixation hépatique). La TDM sans injection de produit de contraste iodé n'a pas été intégrée dans les critères d'interprétation. Les images de fusion ont permis de localiser les foyers hyperfixants.

De façon rétrospective, un calcul du SUVmax correspondant à l'hyperfixation observée pour chaque prothèse a été effectué. Il a été comparé au SUVmax d'une région d'intérêt de référence sur l'aorte abdominale dans le plan des pédicules rénaux (rapport SUV max PV/SUVmax Aorte).

\subsubsection{Scintigraphie aux leucocytes marqués (SLM)}

L'interprétation a été basée sur une analyse visuelle de la distribution des leucocytes marqués. L'aspect de l'hyperfixation des leucocytes marqués (focale, diffuse ou mixte) et l'évolution de son intensité entre les images de six heures et celles de 24 heures (diminuée, stable ou augmentée) ont été appréciés. L'analyse tomoscintigraphique (TEMP) couplée à la TDM réalisée à six heures a permis une localisation précise des foyers hyperfixants visualisés sur les clichés planaires. Comme en TEP, la TDM n'a permis qu'un repérage anatomique.

\subsubsection{Interprétation des données}

Les données ont été analysées de façon rétrospective par deux observateurs indépendants (médecins nucléaires) sans connaissance du contexte clinique ni des autres données d'imagerie. Il a été attribué à chaque patient un numéro d'anonymat pour les examens TEP-FDG et SLM. Ceux-ci ont été analysés de façon aléatoire par les observateurs et seuls étaient portés à leur connaissance le nombre et le site des prothèses. En cas de discordance sur le diagnostic final retenu, l'intervention d'un troisième observateur était requise pour trancher en faveur ou non de l'IPV.

$$
R(\%)=\frac{\text { Activité culot leucocytaire }(\mathrm{MBq})}{\text { Activité }(\text { culot leucocytaire }+ \text { surnageant })(\mathrm{MBq})} \times 100
$$




\subsection{Analyse statistique}

Compte tenu du faible échantillon de patients dans notre cohorte, il n'a pas été effectué de test statistique spécifique. Seule la confrontation à l'étalon de référence pour la caractérisation en vrai ou faux positif (VP ou FP) et vrai ou faux négatif (VN ou FN) a été retenue.

\section{Résultats}

Dans cette étude, $22 \mathrm{PV}$ chez 11 patients ont été explorées. Huit PV (sept patients) sur les 14 sites suspectés ont été considérées comme infectées. Pour trois d'entre elles, les données bactériologiques ont été positives : dans deux cas (patients 5 et 11$)$, les germes ont été retrouvés sur pièce opératoire (E. coli et Streptococcus anginosus) et dans un cas (patient 1) sur hémocultures (Staphylocoque aureus MétiS). Pour ce patient 1 (IPV ilio-fémorale gauche), les examens TEP-FDG et SLM étaient concordants et positifs. Deux contrôles TEP à distance et une SLM se sont avérés négatifs après antibiothérapie.

En ce qui concerne les cinq autres IPV (quatre patients) sans germe retrouvé, le contexte clinique a permis aux cliniciens de trancher devant la présence d'abcès périprothétiques chez le patient 4 (contrôle à neuf mois de la SLM qui s'est négativée après traitement), ainsi que chez les patients 7 et 9 , et de métastases septiques cérébrales chez le patient 6. Ces données sont résumées dans le Tableau 1.

Les résultats des analyses des examens TEP-FDG et SLM par les observateurs sont reportés dans le Tableau 2. Le troisième observateur est intervenu pour l'interprétation des examens TEP-FDG dans un seul cas (patient 8 pour sa PV aortofémorale droite) et dans six cas pour la SLM (patients 1, 3, 5, 6, 7 et 11$)$.

Dans le diagnostic d'IPV, sur les 22 PV, l'examen TEP-FDG a montré $8 \mathrm{VP}, 13 \mathrm{VN}, 1 \mathrm{FP}$ et $0 \mathrm{FN}$ contre $8 \mathrm{VP}, 14 \mathrm{VN}, 0 \mathrm{FP}$ et 0 FN pour la SLM (Tableaux 3 et 4 ). Il n'a pas été retrouvé d'IPV sur les prothèses non suspectes d'infection initialement.

Si l'on considère l'ensemble des $22 \mathrm{PV}$, les résultats sont concordants dans 21 cas avec huit concordances positives et 13 concordances négatives (Tableau 5).

Seul un cas de faux positif (patient 7) a été retrouvé et uniquement en TEP-FDG (1 VN en SLM). Chez ce patient, on retrouvait une hyperfixation intense (supérieure à la fixation hépatique) de ses PV aorto-bifémorales, diffuse à gauche et mixte à droite. L'IPV a été retenue à droite. Ce patient polyvasculaire, opéré plusieurs fois du scarpa droit, était surveillé pour des faux anévrysmes anastomotiques et des lymphocèles bilatéraux. La première chirurgie avait été réalisée

Tableau 3

Tableau de contingence du TEP-FDG et de l'infection de prothèse vasculaire. Contingency table of FDG-PET in vascular prostheses infections.

\begin{tabular}{lllr}
\hline & Infection $(+)$ & Infection $(-)$ & 9 \\
\hline TEP+ & $8(\mathrm{VP})$ & $1(\mathrm{FP})$ & 13 \\
TEP - & $0(\mathrm{FN})$ & $13(\mathrm{VN})$ & 22 \\
& 8 & 14 &
\end{tabular}

$\mathrm{VP}$ : vrai positif ; VN : vrai négatif ; FP : faux positif ; FN : faux négatif.
Tableau 4

Tableau de contingence de la scintigraphie aux leucocytes marqués et de l'infection de prothèse vasculaire.

Contingency table of 99mTc-HMPAO-labeled leukocytes scintigraphy with SPECT-CT in vascular prostheses on infections.

\begin{tabular}{lllr}
\hline & Infection $(+)$ & Infection $(-)$ & 8 \\
\hline Leucocytes+ & $8(\mathrm{VP})$ & $0(\mathrm{FP})$ & 14 \\
Leucocytes - & $0(\mathrm{FN})$ & $14(\mathrm{VN})$ & 22 \\
& 8 & 14 & \\
\hline
\end{tabular}

$\mathrm{VP}$ : vrai positif ; VN : vrai négatif ; $\mathrm{FP}$ : faux positif ; $\mathrm{FN}$ : faux négatif.

en 1999 (pontage aorto-bifémoral). La prothèse à gauche avait donc environ neuf ans au moment de l'examen TEP. Les images observées en TEP et SLM sont représentées sur les Fig. 1 et 2.

Les aspects des anomalies de fixation observées en TEPFDG et SLM suivant le diagnostic retenu ou non d'IPV sont décrits dans les Tableaux 6 et 7 .

Concernant la description en TEP-FDG, les huit cas d'IPV ont été décrits par une hyperfixation focale (quatre cas) ou mixte (quatre cas) d'intensité 3 ou 4 (égale ou supérieure à la fixation hépatique). Hormis le FP et le cas d'infection des parties molles (patient 3), les trois cas de VN ont présenté une hyperfixation diffuse d'intensité 2 (supérieure au bruit de fond (BDF) local mais inférieure à la fixation hépatique).

Pour la SLM, les huit cas d'IPV ont été décrits par une hyperfixation focale stable (un cas) ou augmentée (six cas) à 24 heures et une hyperfixation mixte augmentée à 24 heures (un cas). Les six cas négatifs pour l'IPV ont été présentés comme une absence d'hyperfixation significative dans trois cas, une hyperfixation focale (un cas), diffuse (un cas) ou mixte (un cas), diminuant dans les trois cas à 24 heures.

Seuls trois patients ont pu bénéficier d'une imagerie de contrôle à distance : deux TEP-FDG de contrôle et une SLM pour le patient 1, une TEP-FDG et une SLM pour le patient 2 et une SPNM pour le patient 4 . La TEP-FDG réalisée 15 jours après la reprise chirurgicale pour le patient 9 a été réalisée dans le cadre du bilan d'extension d'une néoplasie bronchopulmonaire.

\section{Discussion}

Les facteurs favorisants les IPV peuvent être opératoires (contexte d'urgence, perforation intestinale) ou postopératoires (infection digestive ou cutanée à distance, thrombose de prothèse, réintervention précoce...). Les patients présentant une immunodépression, une néoplasie, un diabète, sont plus à risque de développer une IPV. Celle-ci nécessite une prise en charge précoce et un traitement lourd chez ces patients souvent polyvasculaires. Elle exige, dans la plupart des cas, l'exérèse de tout le matériel infecté suivie ensuite d'une revascularisation itérative in situ (utilisant les axes artériels primitifs, des autogreffons artériels ou veineux, des allogreffes artérielles ou, dans une moindre mesure, des prothèses imprégnées d'antibiotiques) ou extra-anatomique (pontages extra-anatomiques avec des trajets souvent longs et de moins bonnes performances hémodynamiques, de moins en moins réalisés), ainsi que d'une antibiothérapie si possible ciblée et bactéricide d'au moins 45 jours $[19,20]$. Le scanner peu spécifique en postopératoire 
Tableau 5

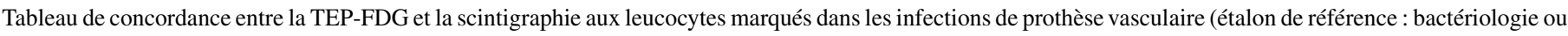
suivi clinicobiologique $>6$ mois).

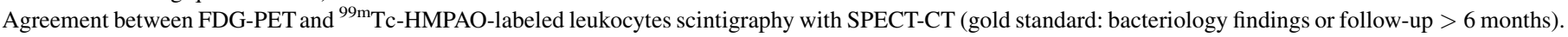

\begin{tabular}{|c|c|c|c|c|c|c|}
\hline Total & $2 \mathrm{VP}$ & $2 \mathrm{VN}$ & VP pour TEP & VP pour SLM & VN pour TEP & VN pour SLM \\
\hline$n=22$ & 8 & 13 & 0 & 0 & 0 & 1 \\
\hline
\end{tabular}

$\mathrm{VP}$ : vrai positif ; VN : vrai négatif ; FP : faux positif ; FN : faux négatif.

est également peu sensible dans les infections pauci-symptomatiques [1,21]. La présentation clinique est souvent fruste quand la prothèse est centrale (aortique ou iliaque), associant des signes généraux aspécifiques (fièvre, sepsis) ainsi qu'un syndrome inflammatoire biologique. Elle est parfois révélée par une hémorragie digestive lors d'une fistule prothéto-digestive. Si la prothèse est périphérique, le diagnostic sera souvent facilité par la présence d'une douleur locale, d'une fistulisation à la peau, d'une lymphorée ou d'un faux anévrisme anastomotique. Moins fréquemment, l'IPV peut se révéler par des métastases infectieuses engendrant endocardites, arthrites, pseudo-panaris septiques, abcès cérébraux [3]. L'apport de l'imagerie métabolique dans le diagnostic de l'IPV est donc important à déterminer afin d'améliorer la prise en charge de ces patients.

\subsection{Critères d'interprétation}

Les critères d'interprétation de la SLM sont relativement " codifiés » et peuvent s'appliquer à n'importe quelle recherche de processus septique en général et notamment

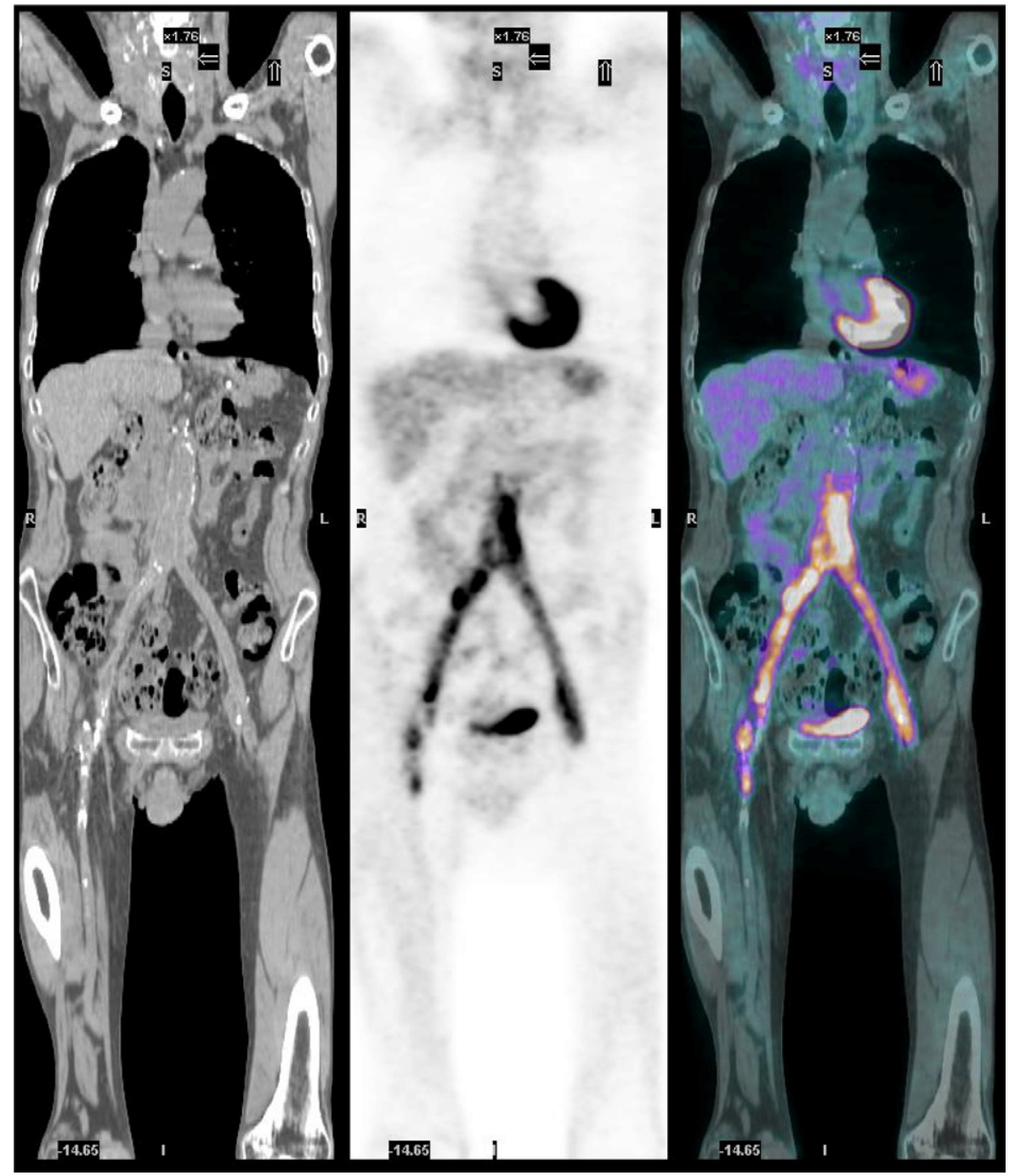

Fig. 1. Fusion coronale TEP-FDG/TDM. Hyperfixation intense (supérieure à la fixation hépatique) des prothèses vasculaires aorto-bifémorales, diffuse à gauche, (FP) et mixte à droite (VP). (patient 7).

FDG-PET/CT coronal fusion. Intense FDG uptake higher than hepatic uptake of vascular aortobifemoral prostheses aortobifemoral, diffuse on the left side (false positive) and mixed on the right side (true positive) (patient 7). 

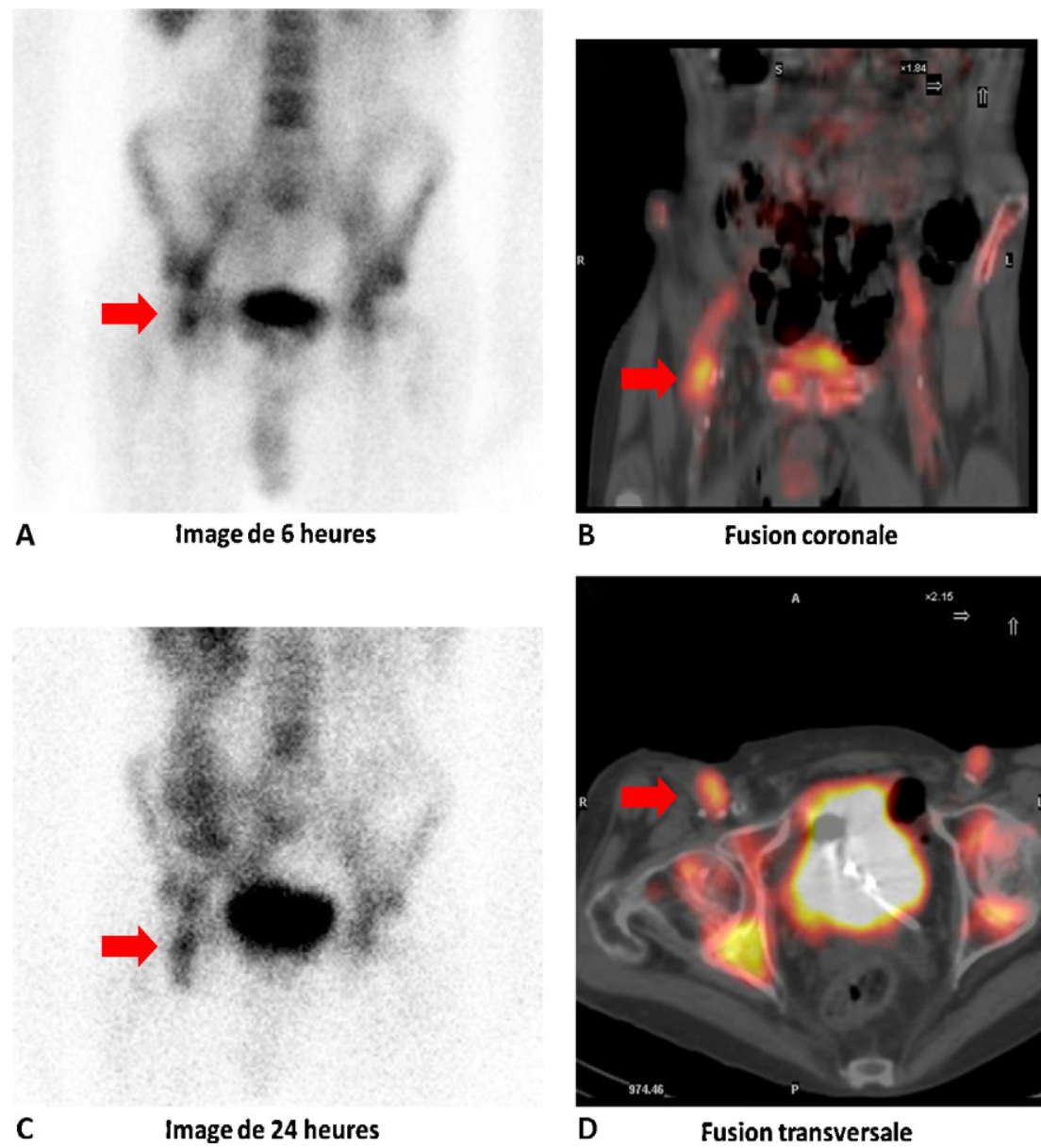

B

Fusion coronale

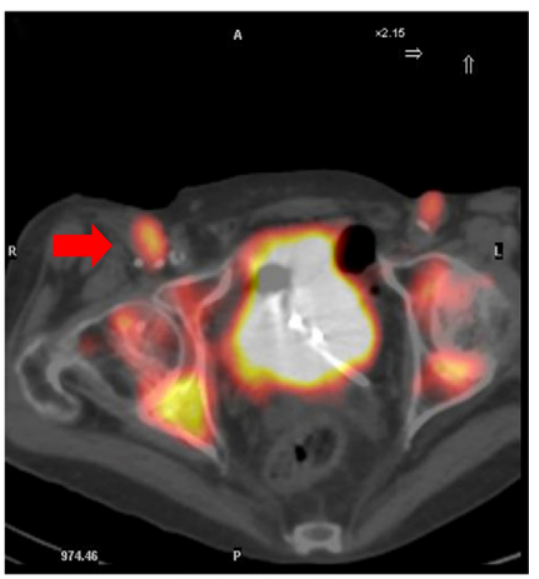

D

Fusion transversale

Fig. 2. Scintigraphie aux leucocytes marqués, clichés planaires (A-C) avec un foyer d'hyperfixation se renforçant entre la sixième et la $24^{\mathrm{e}}$ heure (flèches rouges). Absence d'hyperfixation à gauche. Fusion TEMP-TDM (B-D) : foyer d'hyperfixation situé sur le trajet de la prothèse fémorale droite (patient 7).

Labeled leukocytes scintigraphy planar scans $(A-C)$ displaying a focal prosthetic uptake increasing at the 24th hour (red arrow). Absence of focal uptake on the left side. SPECT-CT fusion $(B-D)$ : focal prosthetic uptake on the right side (patient 7).

pour l'IPV [3]. Le principe repose sur la migration des leucocytes (et notamment des polynucléaires) par chimiotactisme vers les sites inflammatoires et infectieux. Le turn-over des leucocytes est plus lent en cas d'infection, ce qui la distingue de l'inflammation. Habituellement, on retiendra le diagnostic d'infection devant un foyer d'hyperfixation des leucocytes stable ou se rehaussant entre les images de six heures et 24 heures. Cet aspect correspond aux images observées dans notre étude pour les huit VP en SLM (Tableau 6).

Tableau 6

Caractéristiques des prothèses suspectes d'infection à la scintigraphie aux leucocytes marqués

Characteristics of suspicious infected prostheses on 99mTc-HMPAO-labeled leukocytes scintigraphy.

\begin{tabular}{|c|c|c|c|c|}
\hline & & $\mathrm{IPV}+$ & IPV- & Total \\
\hline \multirow[t]{10}{*}{ Hyperfixation leucocytes marqués } & Absente & 0 & 3 & 3 \\
\hline & Focale $>24 \mathrm{~h}$ & 6 & 0 & 6 \\
\hline & Focale $=24 \mathrm{~h}$ & 1 & 0 & 1 \\
\hline & Focale $<24 \mathrm{~h}$ & 0 & 1 & 1 \\
\hline & Diffuse $>24 \mathrm{~h}$ & 0 & 0 & 0 \\
\hline & Diffuse $=24 \mathrm{~h}$ & 0 & 0 & 0 \\
\hline & Diffuse $<24 \mathrm{~h}$ & 0 & 1 & 1 \\
\hline & Mixte $>24 \mathrm{~h}$ & 1 & 0 & 1 \\
\hline & Mixte $=24 \mathrm{~h}$ & 0 & 0 & 0 \\
\hline & Mixte $<24 \mathrm{~h}$ & 0 & 1 & 1 \\
\hline Total & & 8 & 6 & 14 \\
\hline
\end{tabular}

IPV : infection de prothèse vasculaire. 
Tableau 7

Caractéristiques des prothèses suspectes d'infection en TEP-FDG.

Characteristics of suspicious infected prostheses on in FDG-PET.

\begin{tabular}{llllc}
\hline & & IPV + & IPV - & Total \\
\hline Hyperfixation FDG & Focale (3-4) & 4 & 0 & 4 \\
& Diffuse (2) & 0 & 3 & 3 \\
& Diffuse (3-4) & 0 & 2 & 2 \\
Total & Mixte (3-4) & 4 & $1^{\text {a }}$ & 5 \\
& & 8 & 6 & 14
\end{tabular}

IVP : infection de prothèse vasculaire.

${ }^{\mathrm{a}} \mathrm{VN}$ : infection des parties molles.

En TEP, l'hyperfixation du FDG est liée à l'augmentation de l'activité glycolytique des lymphocytes et des macrophages activés, aussi bien sur les sites infectieux que sur les sites inflammatoires. La distinction entre une inflammation et une infection est donc moins spécifique et il n'existe pas à l'heure actuelle de critères standardisés en TEP-FDG pour l'IPV. L'interprétation des images par nos observateurs s'est basée sur des critères d'analyse visuelle à l'aide d'une échelle de valeurs (4 points), en prenant comme seuil de positivité, la fixation hépatique. Dans d'autres études reprenant les critères visuels (échelle de 3, 4 ou 5 points pour les grosses séries), les références ont été soit l'environnement périprothétique (BDF local), soit la fixation des muscles inactifs, de la graisse ou de la vessie (Tableau 8 ).

Certaines études (Tableau 8) ont proposé des indices de rapport de SUV à partir de régions d'intérêt (ROIs) prothétiques, en prenant comme référence des ROIs moyennées vasculaires (Aorte ou VCI). Dans notre étude, un ratio a été calculé entre le SUVmax prothétique et le SUVmax de l'aorte à l'origine des pédicules rénaux pour les 22 prothèses (Tableau 9). Dans les huit cas où il existait une infection de prothèse, le ratio médian était de 4,25 (Min : 1,9-Max : 8,2). Dans les cas où l'IPV n'a pas été retrouvée, le ratio médian était de 1,35 (Min : 1,0 - Max : 5,1). Même s'il existe une différence entre les médianes de ratios, l'écart de ratios est trop important pour conclure sur une éventuelle valeur seuil. On ne peut donc se satisfaire uniquement d'une analyse semi-quantitative. Il est nécessaire d'inclure l'aspect de la fixation dans l'interprétation. Dans son étude, Spacek et al. établissent à partir d'analyses de courbes ROC une valeur de ratio PV/BDF vasculaire égale à 1,74 , donnant les meilleurs chiffres de sensibilité et spécificité (respectivement 74,5 et $82,9 \%$ ). En prenant comme critère l'analyse visuelle (une hyperfixation focale ou hétérogène du FDG), il atteint des valeurs de sensibilité plus élevées $(98,2 \%)$ avec une discrète diminution de spécificité $(75,6 \%)$ par rapport à l'analyse semi-quantitative.

Spacek et al. [1] ajoutent un autre critère à l'aspect de l'hyperfixation TEP qui est l'irrégularité des bords de la PV en TDM. Ces deux paramètres apparaissent comme les meilleurs critères prédictifs de l'IPV, de façon indépendante. Dans notre étude, l'aspect le plus prédictif d'IPV en TEP-FDG a été la présence d'une hyperfixation focale ou mixte supérieure ou égale à la fixation hépatique (Tableau 4). Nous n'avons pas, par

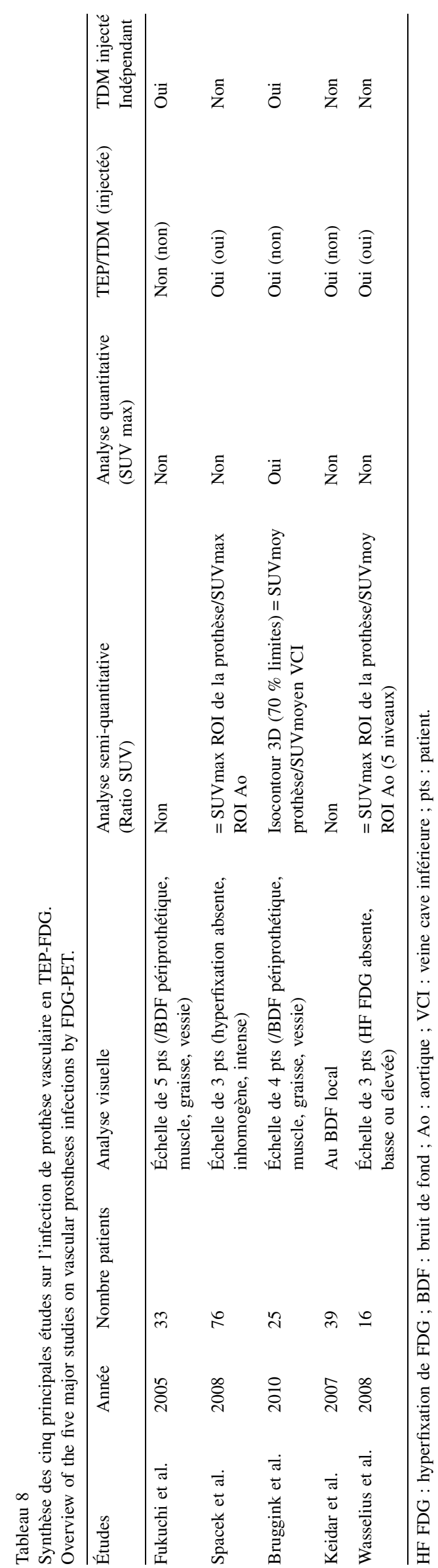


Tableau 9

SUVmax des prothèses vasculaires, ratios SUVmax PV/SUVmax aorte (à hauteur des pédicules rénaux).

Vascular prostheses uptake in FDG-PET, maximum uptake ratios of vascular prosthesis/maximum uptake aorta (renal hiles level).

\begin{tabular}{|c|c|c|c|c|c|}
\hline Numéro patient & Types prothèses & Prothèse (Infection retenue) & SUV max & $\begin{array}{l}\text { Aorte pédicule } \\
\text { rénal SUVmax }\end{array}$ & $\begin{array}{l}\text { Ratio SUVmax } \\
\text { PV/Aorte }\end{array}$ \\
\hline \multirow[t]{2}{*}{1} & \multirow[t]{2}{*}{ Ilio-bifémorales } & G (oui) & 7,3 & 2,1 & 3,5 \\
\hline & & $\mathrm{D}$ (non) & 2,2 & 2,1 & 1,0 \\
\hline 2 & Trépied fémoral D & $\mathrm{D}$ (non) & 2,3 & 1,9 & 1,2 \\
\hline \multirow[t]{2}{*}{3} & \multirow[t]{2}{*}{ Aorto-bifémorales } & $\mathrm{G}$ (non) & 6,3 & 1,9 & 3,3 \\
\hline & & D (non) & 6,3 & 1,9 & 3,3 \\
\hline 4 & Fémoro-poplité G & G (oui) & 5,7 & 1,9 & 3,0 \\
\hline \multirow[t]{2}{*}{5} & \multirow[t]{2}{*}{ By-pass aorto-bifémoral } & G (oui) & 8,5 & 1,7 & 5,0 \\
\hline & & D (non) & 3,1 & 1,7 & 1,8 \\
\hline \multirow[t]{3}{*}{6} & Inter-sous-clavier & 1 (oui) & 16,3 & 2 & 8,2 \\
\hline & Ao thoracique & 1 (non) & 2,8 & 2 & 1,4 \\
\hline & Prothèse tabc & 1 (non) & 2 & 2 & 1,0 \\
\hline \multirow[t]{2}{*}{7} & \multirow[t]{2}{*}{ Aorto-bifémoral } & G (non) & 8,2 & 1,6 & 5,1 \\
\hline & & D (oui) & 10 & 1,6 & 6,3 \\
\hline \multirow[t]{2}{*}{8} & \multirow[t]{2}{*}{ Aorto-fémoral D + iliaque G } & G (non) & 2,6 & 1,4 & 1,9 \\
\hline & & $\mathrm{D}$ (non) & 2,8 & 1,4 & 2,0 \\
\hline \multirow[t]{2}{*}{9} & \multirow[t]{2}{*}{ Ilio-bifémoral } & G (oui) & 9,5 & 1,4 & 6,8 \\
\hline & & D (oui) & 2,6 & 1,4 & 1,9 \\
\hline 10 & Fémoro-poplité G & 1 (non) & 2,2 & 1,8 & 1,2 \\
\hline \multirow[t]{4}{*}{11} & Veine cave inférieure, & VCI (oui) & 5,7 & 1,8 & 3,2 \\
\hline & \multirow[t]{3}{*}{ Aorte sous-rénale, Aorto-bifémorales } & AO sous rénale (non) & 2,3 & 1,8 & 1,3 \\
\hline & & Fémorale D (non) & 1,05 & 1,8 & 0,6 \\
\hline & & Fémorale G (non) & 1,02 & 1,8 & 0,6 \\
\hline
\end{tabular}

$\mathrm{D}$ : droite ; $\mathrm{G}$ : gauche.

ailleurs, intégré la TDM (non injectée) dans nos critères d'interprétation, celle-ci n'ayant qu'une valeur localisatrice (idem en SLM).

\subsection{Concordances entre TEP-FDG et SLM}

Les résultats montrent une bonne concordance entre la TEPFDG et la SLM (Tableau 5) avec cependant une meilleure spécificité de la SLM. Elle constitue dans notre étude l'examen de référence pour prédire l'IPV, alors qu'il existe un cas de FP en TEP-FDG. D' autre part, il n'y a eu aucun cas de faux négatif dans les deux examens. Ce qui fait une sensibilité de 8/8 et une spécificité 14/14 pour la SLM à rapporter à notre faible échantillon de 11 patients. Ces résultats sont nuancés par le fait que l'interprétation des images en SLM s'est avérée plus difficile puisque le troisième observateur a été sollicité dans six cas sur les 14 sites suspectés (cinq cas de VP et un cas de VN) contre un cas en TEP-FDG (un cas de VN). Cela peut être expliqué en partie, par une différence d'expérience des observateurs dans l'interprétation des images en SLM dans l'IPV.

\subsection{Sensibilité-Spécificité de la TEP-FDG}

Concernant nos résultats en TEP-FDG, les valeurs de sensibilité et de spécificité, compte tenu de notre faible échantillon de patients, sont respectivement de 8/8 et de 13/ 14 si l'on considère l'ensemble des 22 PV. Il n'y avait aucun cas d'IPV dans les huit prothèses non suspectes initialement. Notre petit échantillonnage ne nous permet pas de réaliser des analyses statistiques poussées. Le Tableau 10 compare les résultats des différentes publications qui ont étudié l'apport de la TEP-FDG (avec ou sans TDM associée) dans le diagnostic de l'IPV. On remarque que la plupart de ces études sont également constituées de cas clinique(s) ou d'un échantillonnage faible de patients. Seules cinq études (Tableau 8) comportent des cohortes suffisamment importantes pour des analyses statistiques.

\subsection{Faux positifs TEP-FDG}

Parmi ces études, seule celle de Wasselius et al. [22] resten réservée sur les performances de la TEP-FDG. Les auteurs ont réanalysé de façon rétrospective 16 examens TEP-FDG correspondant à des patients porteurs de PV sur 2045 examens réalisés dans leur établissement. Sur ces 16 cas, seul 1 patient présentait une IPV. L'analyse des 15 autres cas (sans IPV) a montré majoritairement une hyperfixation des PV interprétée comme des faux positifs (FP) (1 cas dans notre étude). Wasselius et al. [22] ainsi que Hagerty et al. et Salzmann et al. [23,24] expliquent les faux positifs TEP en évoquant des phénomènes inflammatoires chroniques aseptiques liés à une réaction à corps étranger avec une infiltration périprothétique par des macrophages, des fibroblastes et des cellules géantes. L'importance de ces processus dépendrait du type de PV (phénomènes plus fréquents avec les PV en Dacron : 11/22 PV dans notre étude) et de son site d'implantation (phénomènes plus importants dans les tissus sous-cutanés par rapport aux tissus graisseux) [25].

\subsection{Comparaison TEP-FDG/SLM et apport de la TEMP/ TDM}

Notre étude est pionnière puisqu'elle est la première à comparer sur une même série de patients les performances 
Tableau 10

Synthèse des études au TEP-FDG sur l'infection de prothèse vasculaire.

Overview of studies of vascular prostheses infections on FDG-PET.

\begin{tabular}{|c|c|c|c|c|c|c|c|c|c|c|}
\hline Études & Année & & $\mathrm{Nb}$ patients & Méthodes & \multicolumn{2}{|c|}{ Critères d'interprétation } & VP & $\mathrm{VN}$ & FP & $\mathrm{FN}$ \\
\hline Krupnick et al. & 2003 & & 1 & PET & \multicolumn{2}{|l|}{ Visuel } & 1 & 0 & 0 & 0 \\
\hline Tsunekawa et al. & 2007 & & 1 & PET & \multicolumn{2}{|l|}{ Visuel } & 1 & 0 & 0 & 0 \\
\hline Chacko et al. & 2003 & & 3 & PET & \multicolumn{2}{|l|}{ Visuel } & 2 & 1 & 0 & 0 \\
\hline Lauwers et al. & 2008 & & 5 & PET & \multicolumn{2}{|l|}{ Visuel } & 3 & 2 & 0 & 0 \\
\hline Stumpe et al. & 2000 & & 7 & PET & \multicolumn{2}{|l|}{ Visuel } & 2 & 5 & 0 & 0 \\
\hline Fukuchi et al. & 2005 & & 33 & PET & \multicolumn{2}{|l|}{ Visuel } & 10 & 14 & 8 & 1 \\
\hline Keidar et al. & 2003 & & 1 & $\mathrm{PET} / \mathrm{CT}$ & \multicolumn{2}{|l|}{ Visuel } & 1 & 0 & 0 & 0 \\
\hline Stadler et al. & 2004 & & 1 & $\mathrm{PET} / \mathrm{CT}$ & \multicolumn{2}{|l|}{ Visuel } & 1 & 0 & 0 & 0 \\
\hline Balink et al. & 2007 & & 1 & $\mathrm{PET} / \mathrm{CT}$ & \multicolumn{2}{|l|}{ Visuel } & 1 & 0 & 0 & 0 \\
\hline Tegler et al. & 2007 & & 1 & $\mathrm{PET} / \mathrm{CT}$ & \multicolumn{2}{|l|}{ Visuel } & 1 & 0 & 0 & 0 \\
\hline Jaruskova et al. & 2006 & & 7 & $\mathrm{PET} / \mathrm{CT}$ & \multicolumn{2}{|l|}{ Visuel } & 6 & 0 & 1 & 0 \\
\hline \multirow[t]{2}{*}{ Wasselius et al. } & 2008 & & 16 & $\mathrm{PET} / \mathrm{CT}$ & \multicolumn{2}{|c|}{ Visuel + semi-quantitatif } & 1 & 5 & 10 & 0 \\
\hline & & & & & & $\mathrm{Se}$ & Spe & $\mathrm{V}$ & & VPN \\
\hline Keidar et al. & 2007 & 39 & $\mathrm{PET} / \mathrm{CT}$ & Visuel & & 93 & 91 & 88 & & 96 \\
\hline Spacek et al. & 2009 & 76 & $\mathrm{PET} / \mathrm{CT}$ & Visuel $+\mathrm{s}$ & ntitatif & $78,2-98,2$ & $75,6-92,7$ & & & $76-96,3$ \\
\hline Bruggink et al. & 2010 & 25 & $\mathrm{PET} / \mathrm{CT}$ & Visuel $+\mathrm{s}$ & ntitatif & 93 & 70 & 82 & & 88 \\
\hline
\end{tabular}

VP : vrai positif ; VN : vrai négatif ; FP : faux positif ; FN : faux positif.

diagnostiques de la TEP-FDG et de la SLM (planaires + TEMP-TDM) dans l'IPV. À notre connaissance, on peut recenser un cas clinique publié par Abouhamad et al. [26] où la SLM est réalisée seule (avec TEMP-TDM) sans comparaison à la TEP-FDG, un cas clinique similaire publié par Hofman et al. [27] et enfin un cas clinique publié plus récemment par Gardet et al. [28] comparant la TEP-FDG à la SLM mais sans TEMPTDM.

Si la SLM est considérée comme la technique d'imagerie de référence pour la recherche d'infection, l'essor de l'imagerie hybride va probablement renforcer ce statut. Dans notre étude, tous les patients ont bénéficié en plus des images planaires à la sixième et $24^{\mathrm{e}}$ heures, d'une acquisition TEMP-TDM à la sixième heure, permettant une visualisation du trajet de la PV et la localisation précise du (des) foyer(s) hyperfixant(s). Les Figs. 3 et 4 illustrent les résultats en TEP et en SLM (cas du patient 4). Chez le patient 3, la TEMP-TDM a été déterminante pour diagnostiquer une infection strictement circonscrite aux parties molles, sans atteinte de la PV (Fig. 5).

La SLM reste cependant une technique d'imagerie plus " lourde » dans sa réalisation : nécessité d'un marquage cellulaire occupant le radiopharmacien durant deux à trois heures (possible problème de programmation de l'examen suivant la disponibilité du radiopharmacien), patient convoqué sur deux jours et temps des images long (prétemps de dix minutes à la sixième heure et de 20 minutes à la $24^{\mathrm{e}}$ heure). Dans notre protocole, pour des raisons pratiques et afin de limiter la durée de l'examen, une seule acquisition TEMPTDM a été réalisée à la sixième heure (durée : 40 minutes). Le temps image seul peut être évalué avec un minimum de une heure 30 minutes (deux images planaires étant habituellement nécessaires pour couvrir tout le trajet de la PV). L'avantage de la TEP-FDG est sa facilité de programmation, l'absence de marquage cellulaire par un radiopharmacien et sa durée d'examen plus courte.

Dans son étude portant sur 21 patients, Dumarey et al. [20] proposent pour la recherche d'un processus infectieux de combiner les avantages des deux techniques en réalisant une TEP avec marquage de leucocytes au ${ }^{18}$ FDG. Pour un score visuel supérieur ou égal à 2 (hyperfixation modérée de FDG), sur une échelle allant de 0 à 3 , il obtient une sensibilité de $91 \%$ et une spécificité de $85 \%$. L'absence d'hyperfixation du FDG avait une valeur prédictive négative de $100 \%$. Ces résultats semblent prometteurs et cette technique pourrait avoir un intérêt potentiel dans l'IPV. Cependant, la demi-vie du FDG ne permet pas la réalisation d'images à 24 heures.

\subsection{Biais de l'étude}

Notre étude de faisabilité souffre de plusieurs biais :

- l'absence de critères d'inclusion ou de non inclusion, tous les patients adressés pour suspicion d'IPV dans un contexte aigu ou non ont été explorés en raison de la faible prévalence de cette pathologie ;

- l'intégration par les cliniciens des résultats des examens TEPFDG et SLM initiaux au diagnostic final d'IPV. Cependant, il aurait été difficile sinon illusoire, de ne pas les intégrer compte tenu des implications pronostiques pour le patient, de la non-spécificité de l'imagerie conventionnelle, notamment 


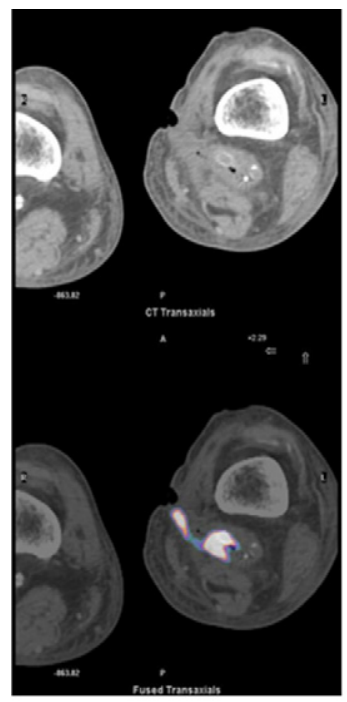

A Fusion transversale

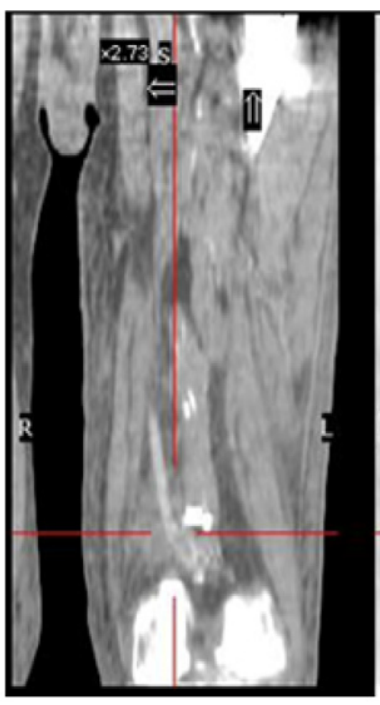

B

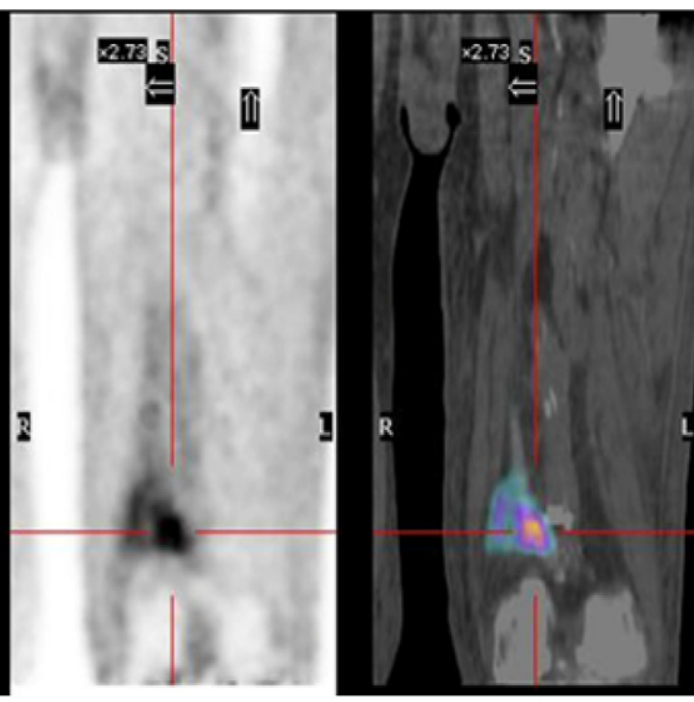

Fusion coronale

Fig. 3. Acquisition TEMP-TDM à la $6^{\text {ème }}$ heure lors de la scintigraphie aux leucocytes marqués, permettant la visualisation du trajet de la prothèse vasculaire fémoro-poplitée gauche et la localisation précise du foyer hyperfixant (patient 4).

SPECT-CT scan at 6th hour, showing a focal increased uptake on the path of left femoropopliteal prostheses with an accurate anatomic localization (patient 4).

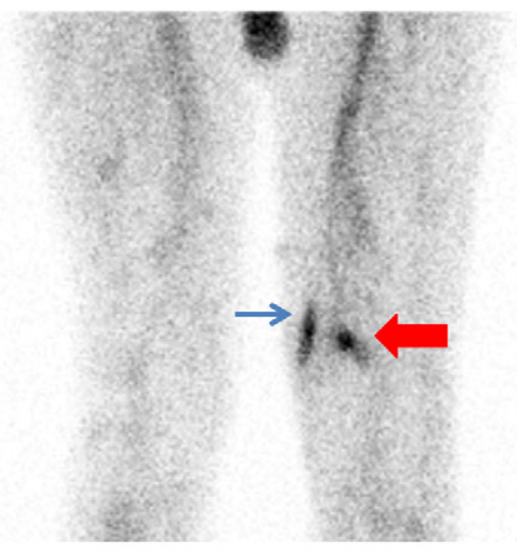

A

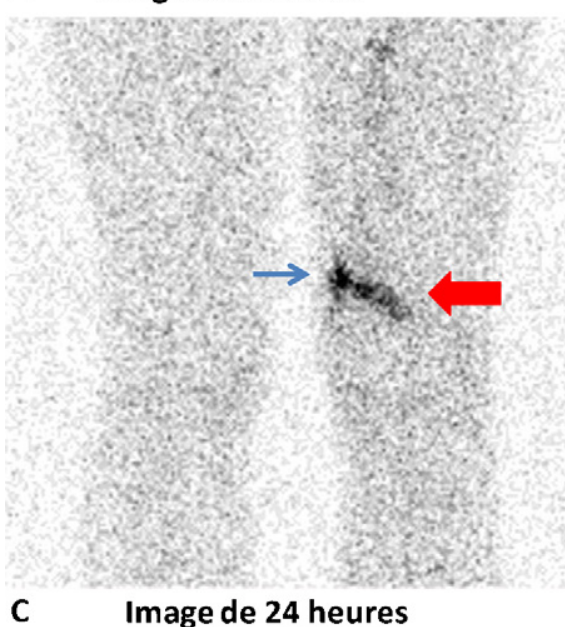

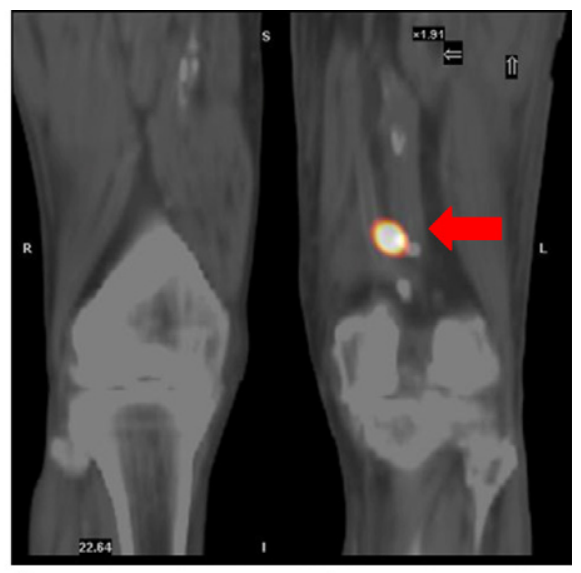

B Fusion coronale

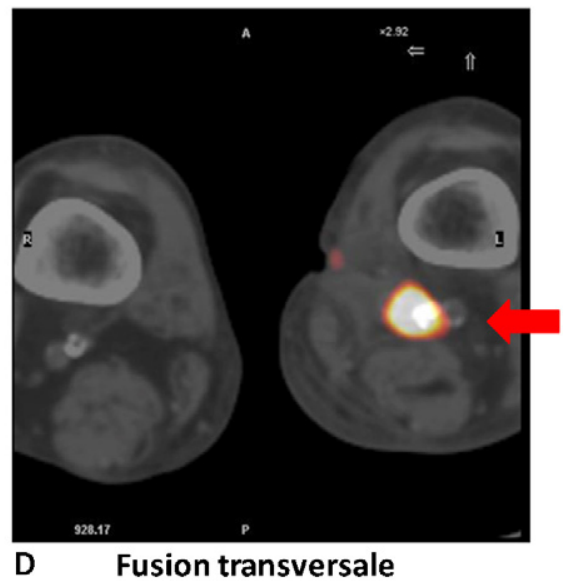

Fig. 4. Clichés planaires (A-C) montrant deux foyers d'hyperfixation se renforçant entre la sixième et la $24^{\mathrm{e}}$ heure (flèche rouge : prothèse-flèche bleue : tissus souscutané) + Acquisition TEMP-TDM à la sixième heure lors de la scintigraphie aux leucocytes marqués, permettant la visualisation du trajet de la prothèse vasculaire fémoro-poplitée gauche et la localisation précise du foyer hyperfixant (patient 4).

Labeled leukocyte scintigraphy planar scans $(A-C)$ showing two focal prosthetic uptake foci increasing at 24th hour (prostheses: red arrow; subcutaneous soft tissues: blue arrow) + SPECT-CT images: accurate localization of the path of the left femoropopliteal prosthesis (patient 4). 


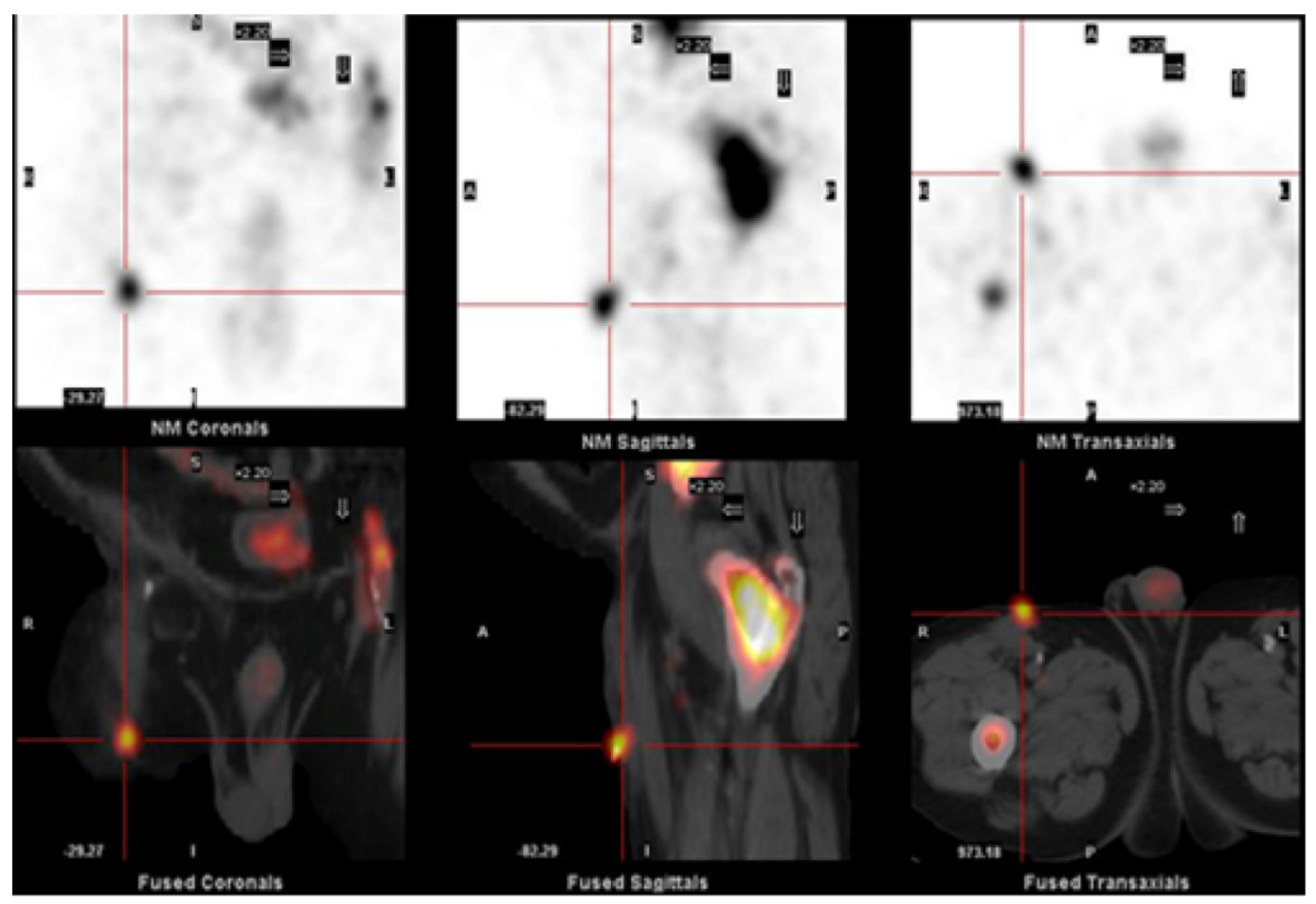

Fusion TEMP-TDM dans les 3 plans

Fig. 5. Scintigraphie aux leukocytes marqués avec TEMP-TDM permettant de diagnostiquer une infection strictement circonscrite aux parties molles, sans atteinte de la prothèse vasculaire (patient 3 ).

Labeled leukocytes scintigraphy with SPECT-CT: allowing to diagnose a soft tissue infection, without vascular prosthesis infection (patient 3).

pour la décision de réintervenir chirurgicalement. Néanmoins, l'analyse rétrospective des données par les deux observateurs s'est faite à l'aveugle, plusieurs mois après la réalisation des examens, sans connaissance des résultats antérieurs et sans connaissance du contexte clinique initial. L'intervention du troisième observateur n'a été sollicitée qu'en cas de discordance des interprétations, également à l'aveugle, l'interprétation majoritaire ayant été retenue ;

- l'hétérogénéité des critères permettant de retenir le diagnostic d'IPV en l'absence de preuve bactériologique directe ; celui-ci résultant d'un faisceau de données clinicobiologiques et d'imagerie ;

- la non réalisation systématique d'examen TEP-FDG et/ou SLM de contrôle pour tous les patients. Seuls trois patients (patients 1,2 et 4) ont bénéficié d'examens à distance et à l'initiative exclusive des cliniciens.

\subsection{Proposition d'arbre décisionnel}

À la suite des résultats préliminaires de notre étude, nous proposons l'attitude suivante dans la recherche d'une IPV : la réalisation en première intention de l'examen TEP-FDG qui est plus facile et plus rapide à organiser. En cas de positivité de l'examen TEP-FDG, avec comme critère la visualisation d'une hyperfixation focale ou mixte de grade 3-4 associée à une probabilité clinique importante, le diagnostic d'IPV peut être fortement suspecté. Si le résultat de l'examen TEP-FDG est litigieux et/ou le contexte clinique moins évident (bactériologie négative notamment, le taux de globules blancs et de CRP n'ayant pas de valeur prédictive significative selon Spacek et al. [1]), une SLM (planaires et TEMP-TDM) peut être demandée en complément afin d'apporter plus d'éléments diagnostiques.

\section{Conclusion}

Peu de séries ont été publiées dans le diagnostic d'IPV par TEP-FDG ou SLM. Les résultats préliminaires de notre étude, dont l'originalité est de comparer ces deux techniques chez les mêmes patients en y associant les avantages de l'imagerie hybride, semblent montrer une excellente sensibilité des deux examens, mais une meilleure spécificité de la SLM avec TEMP couplée à la TDM dans le diagnostic de l'IPV. L'apport de la TEMP-TDM dans la SLM est primordial, car elle permet une évaluation précise de la localisation de l'infection (prothétique ou extra-prothétique) et de son extension.

Cette étude montre la faisabilité et l'intérêt de réaliser une TEP-FDG en première intention dans les suspicions d'IPV, qui est de réalisation plus simple que la SLM, malgré sa moins bonne spécificité. Dans notre étude, un hypermétabolisme focal ou hétérogène supérieur ou égal à la fixation hépatique était prédictif d'une IPV avec une sensibilité à $8 / 8$ et une spécificité de 13/14. Il reste à définir des paramètres semi-quantitatifs (par exemple la valeur seuil du ratio SUVmax prothèse vasculaire/ aorte) permettant d'apporter un argument supplémentaire en faveur du diagnostic. Ces données sont à confirmer par la réalisation d'une étude prospective sur une plus grosse série de patients. Le dernier point serait d'intégrer l'analyse de la TDM 
(avec injection de produit de contraste) afin d'augmenter la valeur prédictive de ces deux examens dans l'IPV [1].

\section{Déclaration d'intérêts}

Les auteurs déclarent ne pas avoir de conflits d'intérêts en relation avec cet article.

\section{Références}

[1] Spacek M, Belohlavek O, Votrubova J, Sebesta P, Stadler P. Diagnostics of "non-acute" vascular prosthesis infection using 18F-FDG PET/CT: our experience with 96 prostheses. Eur J Nucl Med Mol Imaging 2009;36:850-8.

[2] Keidar Z, Engel A, Hoffman A, Israel O, Nitecki S. Prosthetic vascular graft infection: the role of 18F-FDG PET/CT. J Nucl Med 2007;48:1230-6.

[3] Roca M, Martin-Comin J, Becker W, Bernardo-Filho M, Gutfilen B, Moisan A, et al. A consensus protocol for white blood cells labelling with technetium-99 $\mathrm{m}$ hexamethylpropylene amine oxime. International Society of Radiolabeled Blood Elements (ISORBE). Eur J Nucl Med 1998;25:797-9.

[4] Tegler G, Sorensen J, Bjorck M, Savitcheva I, Wanhainen A. Detection of aortic graft infection by 18 -fluorodeoxyglucose positron emission tomography combined with computed tomography. J Vasc Surg 2007;45:82830.

[5] Stadler P, Bilohlavek O, Spacek M, Michalek P. Diagnosis of vascular prosthesis infection with FDG-PET/CT. J Vasc Surg 2004;40:1246-7.

[6] Fukuchi K, Ishida Y, Higashi M, Tsunekawa T, Ogino H, Minatoya K, et al. Detection of aortic graft infection by fluorodeoxyglucose positron emission tomography: comparison with computed tomographic findings. J Vasc Surg 2005;42:919-25.

[7] Van der Vaart MG, Meerwaldt R, Slart RH, van Dam GM, Tio RA, Zeebregts CJ. Application of PET/SPECT imaging in vascular disease. Eur J Vasc Endovasc Surg 2008;35:507-13.

[8] Ducasse E, Calisti A, Speziale F, Rizzo L, Misuraca M, Fiorani P. Aortoiliac stent graft infection: current problems and management. Ann Vasc Surg 2004;18:521-6.

[9] Annovazzi A, Bagni B, Burroni L, D’Alessandria C, Signore A. Nuclear medicine imaging of inflammatory/infective disorders of the abdomen. Nucl Med Commun 2005;26:657-64.

[10] Liberatore M, Iurilli AP, Ponzo F, Prosperi D, Santini C, Baiocchi P, et al. Clinical usefulness of technetium-99m-HMPAO-labeled leukocyte scan in prosthetic vascular graft infection. J Nucl Med 1998;39:875-9.

[11] Prats E, Banzo J, Abos MD, Garcia-Lopez F, Escalera T, Garcia-Miralles $\mathrm{M}$, et al. Diagnosis of prosthetic vascular graft infection by technetium99m-HMPAO-labeled leukocytes. J Nucl Med 1994;35:1303-7.

[12] Filippi L, Schillaci O. Usefulness of hybrid SPECT/CT in $99 \mathrm{mTc}-$ HMPAO-labeled leukocyte scintigraphy for bone and joint infections. J Nucl Med 2006;47:1908-13.
[13] Bar-Shalom R, Yefremov N, Guralnik L, Keidar Z, Engel A, Nitecki S, et al. SPECT/CT using $67 \mathrm{Ga}$ and $111 \mathrm{In}$-labeled leukocyte scintigraphy for diagnosis of infection. J Nucl Med 2006;47(4):587-94, PMID:16595491.

[14] Love C, Marwin SE, Tomas MB, Krauss ES, Tronco GG, Bhargava KK, et al. Diagnosing infection in the failed joint replacement: a comparison of coincidence detection 18F-FDG and 111 In-labeled leukocyte/99mTcsulfur colloid marrow imaging. J Nucl Med 2004;45:1864-71.

[15] Jaruskova M, Belohlavek O. Role of FDG-PET and PET/CT in the diagnosis of prolonged febrile states. Eur J Nucl Med Mol Imaging 2006;33:913-8.

[16] Oyen WJ, Mansi L. FDG-PET in infectious and inflammatory disease. Eur J Nucl Med Mol Imaging 2003;30:1568-70.

[17] Burroni L, D'Alessandria C, Signore A. Diagnosis of vascular prosthesis infection: PET or SPECT? J Nucl Med 2007;48:1227-9.

[18] de Vries EF, Roca M, Jamar F, Israel O, Signore A. Guidelines for the labelling of leucocytes with $(99 \mathrm{~m}) \mathrm{Tc}-\mathrm{HMPAO}$. Inflammation/Infection Taskgroup of the European Association of Nuclear Medicine. Eur J Nucl Med Mol Imaging 2010;37:842-8.

[19] Koskas F, Plissonnier D, Bahnini A, Ruotolo C, Kieffer E. Vascular allografts. Application to the treatment of aorto-iliac prosthetic infections with in situ revascularization. Chirurgie 1997;122:13-7.

[20] Dumarey N, Egrise D, Blocklet D, Stallenberg B, Remmelink M, del Marmol V, et al. Imaging infection with 18F-FDG-labeled leukocyte PET/ CT: initial experience in 21 patients. J Nucl Med 2006;47:625-32.

[21] Low RN, Wall SD, Jeffrey Jr RB, Sollitto RA, Reilly LM, Tierney Jr LM. Aortoenteric fistula and perigraft infection: evaluation with CT. Radiology 1990;175:157-62.

[22] Wasselius J, Malmstedt J, Kalin B, Larsson S, Sundin A, Hedin U, et al. High 18F-FDG Uptake in synthetic aortic vascular grafts on PET/CT in symptomatic and asymptomatic patients. J Nucl Med 2008;49:1601-5.

[23] Hagerty R, Salzmann DL, Kleinert LB, Williams SK. Cellular proliferation and macrophage populations associated with implanted expanded polytetrafluoroethylene and polyethyleneterephthalate. J Biomed Mater Res 2000;49(4):489-97, PMID:10602082.

[24] Salzmann DL, Kleinert LB, Berman SS, Williams SK. Inflammation and neovascularization associated with clinically used vascular prosthetic materials. Cardiovasc Pathol 1999;8:63-71.

[25] van Bilsen PH, Popa ER, Brouwer LA, Vincent J, Taylor CE, de Leij LF, et al. Ongoing foreign body reaction to subcutaneous implanted (heparin) modified Dacron in rats. J Biomed Mater Res A 2004;68:423-7.

[26] Abouhamad P, Krug B, Picard C, Nogarede C, Seldrum M, Buche M, et al. Improved diagnosis of aortobifemoral vascular graft infection by In-111labeled leukocyte SPECT. Clin Nucl Med 2006;31:338-9.

[27] Hofmann A, Zettinig G, Wachter S, Kurtaran A, Kainberger F, Dudczak R. Imaging of aortic prosthesis infection with a combined SPET/CT device. Eur J Nucl Med Mol Imaging 2002;29:836.

[28] Gardet E, Addas R, Monteil J, Le Guyader A. Comparison of detection of F-18 fluorodeoxyglucose positron emission tomography and $99 \mathrm{mTc}-$ hexamethylpropylene amine oxime labelled leukocyte scintigraphy for an aortic graft infection. Interact Cardiovasc Thorac Surg 2010;10:142-3. 